\title{
Lymphocytic expression of Fas and FasL apoptotic markers in vitiligo
}

\author{
Fatma Y. Saleh, Sherif Shoukry Awad", Irene M. Sadek \\ Department of Dermatology and Venereology, Faculty of Medicine, Minia University, Egypt
}

Email address:

sherifu@rocketmail.com(S. S. Awad)

\section{To cite this article:}

Fatma Y. Saleh, Sherif Shoukry Awad, Irene M. Sadek. Lymphocytic Expression of Fas and FasL Apoptotic Markers in Vitiligo. Clinical Medicine Research. Vol. 2, No. 5, 2013, pp. 105-109. doi: 10.11648/j.cmr.20130205.13

\begin{abstract}
Background. Lymphocytic dermal infiltrations accompany the melanocytes loss in depigmenting vitiligo skin. These lymphocytes are incriminated during the pathogenesis of the disease. Fas receptor is a death receptor on the surface of cells that leads to programmed cell death (apoptosis) and FasL is its ligand. Fas/FasL system plays a crucial role in modulating apoptosis. Objectives. This study investigates the lymphocytic expression of apoptotic markers Fas/FasL in vitiligo. Methods. The present study was conducted on 45 vitiligo samples obtained from 15 vitiligo patients including 8 females $(53.3 \%)$ and seven males (46.7\%). Biopsies were obtained also from control volunteers. All specimens were routinely stained with Hematoxylin \&Eosin and immunohistochemically for Fas \& FasL apoptotic markers. Results. Fas \& FasL were significantly expressed by infiltrating lymphocytes in vitiligo biopsies compared to control. Lymphocytic expression of Fas \& FasL was higher on the edge biopsies than the center. Considerable lymphocytic infiltration and attack of B.M. were associated with higher lymphocytic Fas and FasL expression. During vitiligo activity lymphocytic Fas \& FasL expression was also significantly higher. Conclusions. Lymphocytic Fas and FasL are significantly expressed in vitiligo patches which may trigger epidermal apoptosis and loss of melanocytes. At the same time these factors have a role in switching-off of the immune responses and cell mediated cytotoxicity.
\end{abstract}

Keywords: Vitiligo, Fas, FasL, Apoptosis

\section{Introduction}

Vitiligo is a complex disease in which depigmentation results from autoimmune loss of melanocytes from affected regions ${ }^{1}$. The progressive loss of melanocytes from vitiligo skin is usually accompanied by cellular infiltrates containing cytotoxic $T$ cells that are suspected in the pathogenesis of the disease as they are able to eradicate pigment cells ${ }^{2,3}$.

Apoptosis stimulation Fragment (Fas) and its ligand (FasL) are typical members of the tumor necrosis factor (TNF) receptor and TNF ligand family respectively, with a pivotal role in the regulation of apoptotic processes ${ }^{5}$. Fas receptor is a death receptor on the surface of cells that leads to programmed cell death (apoptosis). It is one of two apoptosis pathways, the other being the mitochondrial pathway. Fas is also known as CD95 and Apo- $1^{6}$. FasL is a well-known inducer of apoptosis in cells expressing its receptor $\mathrm{Fas}^{7}$.

Several studies have demonstrated that the Fas and FasL abnormalities are associated with an increased risk of many autoimmune diseases and might be associated with risk of vitiligo too ${ }^{8}$.

This work aims to evaluate the expression of Fas and FasL by dermal lymphocytic infiltrates in vitiligo, possibly to explain their role during disease development.

\section{Methods}

The study was conducted on 45 samples obtained from 15 non segmental vitiligo patients presented to the outpatient dermatology clinic of university hospital and 4 normal non-vitiligo volunteer control subjects after obtaining their consent and approval of the local ethical committee.

All patients were subjected to: history taking, clinical examination and skin biopsy. Three biopsies were taken from each patient; 2 from vitiliginous patch; one at the edge and the other at the center and 1 biopsy from non vitiliginous skin in gluteal region. Three millimeter punch probes were used to obtain all samples after local anesthesia. Another 4 biopsies were included from the normal volunteer persons.

Biopsies were fixed in $10 \%$ formalin, embedded in paraffin blocks then sectioned at $5 \mu \mathrm{m}$ thickness, mounted 
on glass slides to be subjected to histopathological examination after hematoxylin \& eosin (H\&E) and immunohistochemical staining for Fas \& FasL.

All biopsies were stained according to manufacturer instruction with monoclonal mouse anti human Fas antibody (code no.: MS-1098-SO, NeoMarkers, Fremont, CA, USA) at a dilution 1:30, and Rabbit Polyclonal FasL antibody (code no.: RB-9029-PO, NeoMarkers, Fremont, CA, USA) at a dilution 1:50. UltraVision LP detection system HRP Polymer \& AEC Chromogen (code no.: TK-015-HA, thermo scientific, CA, USA) was used as high efficiency detection system which is known as the latest technology in polymeric labeling and counterstained in hematoxylin.

Positive controls from small intestine (strong positive Fas reactivity) and tonsils (strong positive FasL reactivity) were included during immunohistochemical staining. Positive cellular localization is cell membrane and cytoplasmic for both Fas and FasL.

Computerized light microscope with a built-in camera (Accu-Scope \#3025 -OLYMPUS) was used to examine and photograph all sections. Fas and FasL expression was evaluated after examining at least 7 different high power fields (HPF) of each section. Positivity was considered when characteristic red staining is obtained at membrane or cytoplasmic portion of lymphocytes in more than one HPF.

Statistical analysis was carried out using statistical package for the social sciences (SPSS, Version 13.0.1). Quantitative data were analyzed as mean \pm standard deviation (SD), while qualitative data were analyzed as frequency distribution test. $Z$ test (test of proportion) was used to detect significant difference of frequency proportion between studied cases. Probability (p) value $<0.05$ was considered statistically significant.

\section{Results}

Eight female patients (53.3\%) and seven male (46.7\%) were included in this study. The age of these patients ranged from 12 to 63 years (mean 33.5 years $\pm 13.7 \mathrm{SD}$ ). Onset of the disease ranged from 1 month to 21 years (mean 68.09 months $\pm 65.6 \mathrm{SD}$ ). Appearance of new lesions ranged from one to 96 weeks (mean 24.1 weeks $\pm 17.7 \mathrm{SD}$ ). Seven patients (46.7\%) were under treatment at the time of biopsy. A positive family history presented in 3 patients (20\%). Five patients $(33.3 \%)$ had activity during last month before study (Table 1).

Routine H\&E histopathological examination of patients biopsies revealed considerable dermal mononuclear infiltration in $14(93.3 \%)$ of biopsies at the edge, $11(73.3 \%)$ of biopsies at the center and $7(46.7 \%)$ biopsies at normal skin. A statistically significant difference was found on comparing presence of infiltration in normal skin with edge; $\mathrm{p}$ value $=0.007$. On the edge, lymphocytic attack of basement membrane was demonstrated in $14(93.3 \%)$ biopsies, $10(66.7 \%)$ biopsies at the center and $3(20 \%)$ biopsies of normal skin. A statistically significant difference was found on comparing presence of attack of basement membrane in normal skin with the edge; $\mathrm{p}$ value $=0.001$.

Table (1): Data of the patients

\begin{tabular}{lll}
\hline Data & \multicolumn{2}{l}{ Total no=15 } \\
\hline \multirow{2}{*}{ Sex } & Male & $7(46.7 \%)$ \\
& Female & $8(53.3 \%)$ \\
Age (years) & Range & $12-63$ \\
& Mean \pm SD & $33.5 \pm 13.7$ \\
Disease onset & Range & $1-252$ \\
(months) & Mean \pm SD & $68.09 \pm 65.6$ \\
& Range & $1-96$ \\
Appearance of new lesions (weeks) & Mean \pm SD & $24.1 \pm 17.7$ \\
& treatment & $7(46.7 \%)$ \\
Treatment & No & $8(53.3 \%)$ \\
& Positive & $3(20 \%)$ \\
Family history & Negative & $12(80 \%)$ \\
\hline
\end{tabular}

Immunohistochemistry results (Table 2, Fig. 1, 2) revealed Fas lymphocytic expression in 10 out of 15 biopsies (66.7\%) on the edge. Positive on the center in 6 biopsies (40\%), while in normal skin was positive in one biopsy (6.7\%). A statistical significant difference was found on comparing Fas positivity in normal skin with edge and center; $\mathrm{p}$ value $=$ 0.003 and 0.01 respectively. Lymphocytic expression of FasL was detected in all biopsies $(100 \%)$ on the edge, while positive on the center in 13 biopsies $(86.7 \%)$. In normal skin was positive in 2 biopsies (13.3\%). A statistically significant difference was found on comparing FasL positive expression in normal skin with edge and the center; $p$ value $=0.008$ and 0.005 respectively. On the edge, 10 patients $(66.7 \%)$ who were positive for FasL were also positive for Fas while in the center only 5 patients were positive for Fas \& FasL. This was significant with $\mathrm{p}$ value $=0.03$.

Table (2): Expression of Fas and FasL apoptotic markers in lymphocytic infiltrates in vitiligo and their relation to disease activity.

\begin{tabular}{lllllll}
\hline & & & & & \\
& & & & & \\
\end{tabular}




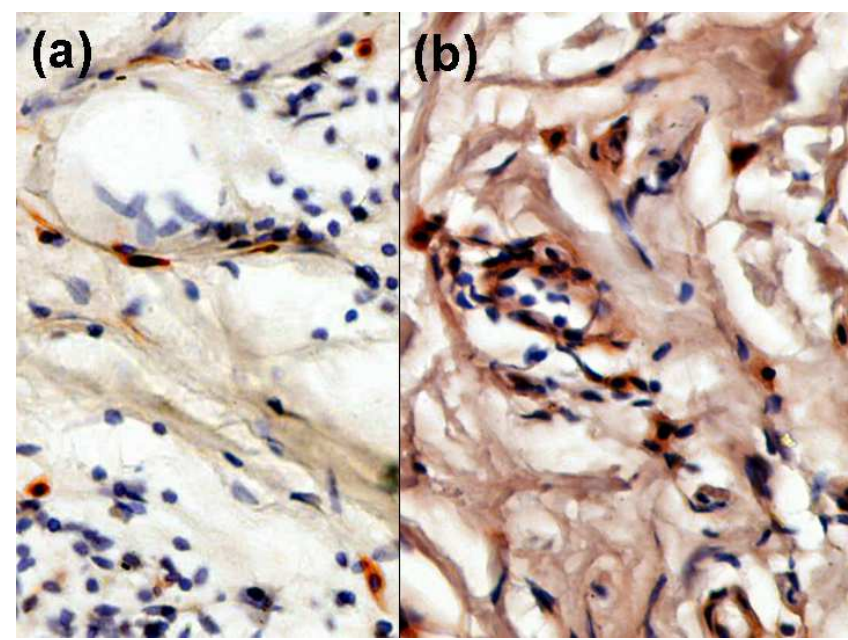

Figure (1): Characteristic membrane and cytoplasmic red staining of lymphocytes expressing apoptotic marker Fas (a) and FasL (b) within infiltrates of vitiligo lesions (Fas and FasL immune-staining, x400).

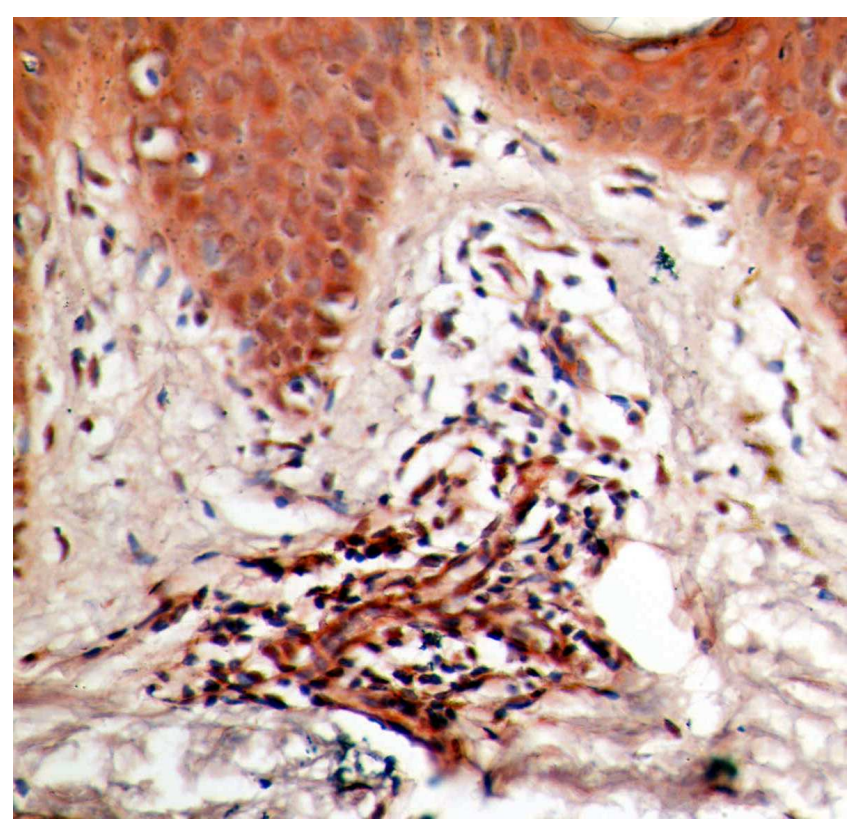

Figure (2): Significant lymphocytic infiltration positively stained for FasL apoptotic marker on edge biopsy of active vitiligo. (FasL immune-staining, x200).

The association between attack of basement membrane (BM) and Fas expression existed on the edge in 9 biopsies $(60 \%)$, on the center in 2 biopsies $(13.3 \%)$ and was completely absent in normal skin, while the association between attack of BM and FasL expression was on the edge in 14 biopsies $(93.3 \%)$, on the center in 8 biopsies $(53.3 \%)$ and in normal skin in 2 biopsies (13.3\%). More significant FasL expression was demonstrated and correlated to BM attack. Again, statistical significant difference was found on comparing edge and center regarding the association between attack of BM \& Fas and attack of BM \& FasL expression with $\mathrm{P}$ value $=0.003$ and 0.006 respectively.

The association between the existence of considerable dermal infiltration and Fas expression was on the edge in 9 biopsies $(60 \%)$ and on the center in 3 biopsies $(20 \%)$, while the association between the considerable infiltration and FasL expression was on the edge in 14 biopsies (93.3\%), on the center in 10 biopsies $(66.6 \%)$ and normal skin in only one biopsy $(6.7 \%)$.

Biopsies of the 5 active vitiligo patients showed attack of $\mathrm{BM}$ in $100 \%$ on edge and center and only in $40 \%$ of normal skin. Also there was considerable lymphocytic infiltration in all biopsies $(100 \%)$ on edge, $80 \%$ on center and $20 \%$ at normal skin. All 5 active patients on edge $(100 \%)$ were positive for Fas \& FasL, on the center all 5 active patients were negative for Fas but 4 patients $(80 \%)$ were positive for FasL. In normal skin all 5 active vitiligo patients were Fas negative and only one patient $(20 \%)$ was FasL positive (Table 2). Relation between Fas expression at edge and center in activity showed statistical significant with $p$ value $=0.01$.

Positive Fas expression existed on the edge in $71.4 \%$ of patients under treatment and in $62.5 \%$ of patients not under treatment, on the center; in $41.9 \%$ of patients under treatment and $37.5 \%$ of patients not under treatment. Positive FasL expression on the edge was detected in all patients whether under treatment or not under treatment, on the center; in $71.4 \%$ of patients under treatment and $100 \%$ of patients not receiving treatment. No statistically significant difference was found.

All biopsies from non vitiligo volunteer subjects did not show significant pathological changes or relevant lymphocytic infiltrate expressing Fas or FasL.

\section{Discussion}

T-cell-mediated cytotoxicity is a main incriminated element in the development of vitiligo lesions ${ }^{4}$. The significant lymphocytic dermal infiltrates were demonstrated in this study and were abundant near edges of vitiligo lesions, more significantly during disease activity, with less abundance during stability or in center of lesions. Epidermotropism with attack of basement membrane zone were manifested too.

Fas and FasL apoptotic markers were significantly expressed by lymphocytic dermal infiltrates. The edge expression of lymphocytic Fas and FasL was more significant than in the center biopsies. Also more significant expression of Fas and FasL was demonstrated within active lesions when compared to stable cases. Bing et al. previously confirmed similar more Fas expression during vitiligo activity than in stability ${ }^{9}$, although Zhang et al. did not find this difference in peripheral blood T cells ${ }^{10}$.

Our finding of this significant expression was correlated to a significant epidermal BM attack which demonstrates the destructive process during vitiligo that can result from infiltration by activated $\mathrm{T}$ cells ${ }^{11}$.

Fas also has a well known role in induction of lymphocytes, Fas signaling is involved in $\mathrm{T}$ cell co-stimulation and proliferation and Fas-FasL interactions was described to facilitate organ-specific immunopathology ${ }^{12}$. This may explain the existence of this 
significant Fas expression during vitiligo activity and toward its progressing active edges. Fas was demonstrated in all edge biopsies of active cases. This expressed Fas possibly stimulates the induction of cytotoxic lymphocytes leading to further melanocytic destruction.

FasL was also demonstrated in all edge biopsies during vitiligo activity and provided another factor in induction of cytotoxicity and vitiligo progression. The FasL expression was significantly correlated to attack of BM as described before.

Paulsen and Janssen found that high doses of cellular FasL almost completely silence $\mathrm{T}$ cells by blocking early TCR-induced signaling events. In contrast, under otherwise unchanged conditions, lower amounts of the same agonists dramatically augment TCR/CD3-driven activation and proliferation $^{13}$. Accordingly and consistent with our findings, we can postulate that during vitiligo activity low levels of FasL expression may exist in order to enhance $\mathrm{T}$ cell proliferation which is commonly reported on active edges, while during stability the edge lymphocytes may express higher levels of FasL in order to induce apoptosis of lymphocytes themselves. It is worthy to mention that edge lymphocytic FasL expression was found in all studied cases whether in active or stable vitiligo and quantitative analysis of cellular expression is required to confirm this hypothesis.

A mild increase in Fas expression, on edge and center, was noticed in cases receiving vitiligo therapy. This induction of Fas expression may enhance self termination of the immune process. In accordance with Dianzani et al. who concluded that Fas is involved in the switching-off the immune responses and cell mediated cytotoxicity ${ }^{14}$. Ligation of FasL to its Fas receptor can result in cell death to limit uncontrolled T-cell expansion during immune responses to terminate the process ${ }^{15}$.

It is confirmed that $\mathrm{T}$ cells are sufficient to initiate the destruction of melanocytes, in addition to being adept at providing help to other effector lineages. Consequently, $\mathrm{T}$ cells were considered proficient therapeutic targets for vaccination and treatment of spontaneous melanoma and vitiligo $^{16}$. Therapies intended to halt many autoimmune diseases were described to induce apoptotic process within lymphocytes to induce termination of the unwanted immunologic destruction ${ }^{17}$. Similar strategy was utilized by using $\mathrm{T}$ cell inhibitor Leflunomide to halt vitiligo activity in a recent study ${ }^{18}$. On the other pole of the spectrum, it was found that melanoma cells can evade the immune system through down-regulation of Fas ${ }^{16}$ in accordance with our findings and hypothesis.

It was previously stated that abnormal expression of Fas and FasL and functional polymorphisms in their genes might be associated with risk of many autoimmune diseases ${ }^{19,20}$ and recently vitiligo too ${ }^{21}$.

In conclusion, Fas and FasL are significantly expressed by lymphocytes in vitiligo patches. This expression is more significant at the progressing edges and during disease activity. Considerable lymphocytic expression and attack of BM zone accompany this significant expression.
Multifaceted functions of these markers exist, as Fas and FasL can stimulate the proliferation and activation of lymphocytes and enhance disease progression, but on the other hand can induce self termination of the autoimmune process. Levels of expression and/or other factors may trigger the switch on and off between activity and stability of the disease.

\section{References}

[1] Spritz RA. Six Decades of Vitiligo Genetics: Genome-Wide Studies Provide Insights into Autoimmune Pathogenesis. J Invest Dermatol. 2011; 132(2): 268-73.

[2] Oyarbide-Valencia K, van den Boorn JG, Denman CJ, et al.. Therapeutic implications of autoimmune vitiligo $\mathrm{T}$ cells. Autoimmun Rev. 2006; 5(7): 486-92.

[3] van den Boorn JG, Konijnenberg D, Dellemijn TA, et al. Autoimmune destruction of skin melanocytes by perilesional T cells from vitiligo patients. J Invest Dermatol. 2009; 129(9): 2220-32.

[4] Steitz J, Wenzel J, Gaffal E and Tuting T. Initiation and regulation of $\mathrm{CD} 8+\mathrm{T}$ cells recognizing melanocytic antigens in the epidermis: implications for the pathophysiology of vitiligo. Eur J Cell Biol. 2004; 83(11): 797-803.

[5] Wajant H, Pfizenmaier K and Scheurich P. Non-apoptotic Fas signaling. Cytokine Growth Factor Rev. 2003; 14(1): 53-66.

[6] Wajant $\mathrm{H}$. The Fas signaling pathway: more than a paradigm. Science. 2002; 296(5573): 1635-6.

[7] Tarzi RM, Sharp PE, McDaid JP, et al. Mice with defective Fas ligand are protected from crescentic glomerulonephritis. Kidney Int. 2011; 81(2): 170-8.

[8] Li M, Sun D, Li C, et al. Functional polymorphisms of the FAS gene associated with risk of vitiligo in Chinese populations: a case-control analysis. J Invest Dermatol. 2008; 128(12): 2820-4.

[9] Bing X, Hongyang L, Weimin C, et al. Pigmentary disorders. Journal of Dermatology. 2010; 37 (1): 111-8.

[10] Zhang QG, Fang $\mathrm{CH}$, Zeng J, et al. The Fas and FasL expression of peripheral blood $\mathrm{T}$ lymphocytes in the patients with active vitiligo vulgaris. Journal of Clinical Dermatology. 2006; 03: 147-8.

[11] Lee AY, Youm YH, Kim NH, et al. Keratinocytes in the depigmented epidermis of vitiligo are more vulnerable to trauma (suction) than keratinocytes in the normally pigmented epidermis, resulting in their apoptosis. $\mathrm{Br} \mathrm{J}$ Dermatol. 2004; 151(5): 995-1003.

[12] Siegel RM, Chan FK, Chun HJ, Lenardo MJ. The multifaceted role of Fas signaling in immune cell homeostasis and autoimmunity. Nat. Immunol. 2000; 1: 469-74.

[13] Paulsen M and Janssen O. Pro- and anti-apoptotic CD95 signaling in T cells. Cell Commun Signal. 2011; 9: 7-14.

[14] Dianzani U, Chiocchetti A and Ramenghi U. Role of inherited defects decreasing Fas function in autoimmunity. Life Sci. 2003; 72(25): 2803-24. 
[15] Osborn SL, Diehl G, Han SJ, et al. Fas-associated death domain (FADD) is a negative regulator of T-cell receptor-mediated necroptosis. Proc Natl Acad Sci U S A. 2010; 107(29): 13034-9.

[16] Lambe T, Leung JC, Bouriez-Jones T, et al. CD4 T cell-dependent autoimmunity against a melanocyte neoantigen induces spontaneous vitiligo and depends upon Fas-Fas ligand interactions. J Immunol. 2006; 177(5): 3055-62.

[17] Montagna P, Brizzolara R, Soldano S, et al. Sex hormones and leflunomide treatment of human macrophage cultures: effects on apoptosis. Int J Clin Exp Med. 2009; 2(3): 221-32.

[18] Awad SS. Leflunomide is a possible deactivator for vitiligo, a pilot study. J Eur Acad Dermatol Venereol. 2012; 26(9): 1173.

[19] Mullighan CG, Heatley S, Lester S, et al. Fas gene promoter polymorphisms in primary Sjögren's syndrome. Ann Rheum Dis. 2004; 63:98-101.

[20] Nolsøe RL, Kelly JA, Pociot F, et al. Functional promoter haplotypes of the human FAS gene are associated with the phenotype of SLE characterized by thrombocytopenia. Genes Immun. 2005; 6:699-706.

[21] Kim NH, Jeon S, Lee HJ and Lee AY. Impaired PI3K/Akt activation-mediated NF-kappaB inactivation under elevated TNF-alpha is more vulnerable to apoptosis in vitiliginous keratinocytes. J Invest Dermatol. 2007; 127(11): 2612-7. 